\title{
Design of Smart Traffic Light Controller Using Embedded System
}

\author{
Vishakha S.Thakare ${ }^{1}$, Snehal R.Jadhav ${ }^{2,}$ Sananaj G.Sayyed ${ }^{3}$, Poonam V. Pawar ${ }^{4}$ \\ ${ }^{1}$ (Computer Department,, Gokhale Education Societies College Of Engineering, India) \\ ${ }^{2}$ (Computer Department,, Gokhale Education Societies College Of Engineering, India) \\ ${ }^{3}$ (Computer Department, Gokhale Education Societies College Of Engineering, India) \\ ${ }^{4}$ (Computer Department, Gokhale Education Societies College Of Engineering, India)
}

\begin{abstract}
The occurrence of traffic congestion at the intersections in cities is one of major problem. The present traffic light controlling system could not sufficient to tackle the traffic congestion due to the lack of efficiency in the designing. The main design problems of present traffic light controller system are:
\end{abstract}

A. Fixed Time Interval:

Vehicles at the traffic lane wait until the green light on. Due to the fixed time interval of red \& green light, suppose a road is always crowded with vehicles and go-ahead time is short. So, vehicles can't pass through the road in the time allowed. But sub lane has few vehicles and go-ahead time is relatively long. The proposed system provides dynamic time interval for traffic lights according to length of vehicles present at each lane.

\section{B. No mechanism provided for the occurrence of an emergency vehicle:}

The emergency vehicle such as ambulance, fire brigade and police will be stuck especially at the traffic light junction. To solve these problems, we have proposed the system called as "Smart Traffic Light Controlling System". The proposed system handles the occurrence of emergency vehicle. It makes all the signals red except one from the emergency vehicle passes.

\section{No Traffic information to user:}

The proposed system provides GSM phone interface to the user, with SMS facility to those who wish to obtain the latest traffic information on congested roads. The map of the traffic signal will be provided to the users on their request.

The proposed system has been developed using the sensor assembly along with embedded technology.

Keywords -Embedded Technology, GSM phone interface, Sensor assembly, Smart Traffic Light Control.

\section{INTRODUCTION}

The rapid growth in the vehicle ownership is one of the measures for economic growth of country. However indirect effect of vehicle ownership is acute traffic congestion. The exploitation of new trends and technologies requires fast transportation of goods, machinery and manpower for various reasons.

The goal of each one is to reach at destination without wasting time and money. But resources provided by current infrastructures are limited. So the Traffic management at road is crucial to reduce waiting and traveling times, save fuel and money. Even though present traffic light controlling system handles the traffic at intersections, many times congestion, accidents happened due to its poor performance.

Suppose a vehicle has to travel a hundred miles to reach its destination via some city and on its way it has to pass through numerous intersections of city. If the traffic system at those intersections is controlled by preset timers then the vehicle will waste precious time at most intersections.

The expansion of road infrastructure is not ultimate solution to the traffic congestion. It requires some smart mechanism that deals with the problems in the present traffic controlling system. The proposed system also provides the map feature, which shows the traffic situation of requested traffic signal.

The problems in the conventional traffic control system are overcome with the following features of proposed system:

\section{A. Dynamic time interval:}

The present traffic light control system provides fixed time interval for red and green light. This causes unnecessary waiting time. As the design of proposed system provides dynamic traffic light intersection that will minimize the waiting time of vehicles and also manage the traffic load at the intersection adaptively.

This maximizes average number of vehicles passing through each intersection.

\section{B. Emergency vehicle management:}

The priority has been given to emergency vehicle like ambulance, Fire Bridge by glowing red lights to all lanes except from the lane where emergency vehicle is passing. 


\section{GSM mobile Interface:}

The information about congestion on road or possible alternate routes can also be informed to car drivers on demand on his/her GSM mobile phone.

\section{Literature Survey}

In this section, the various solutions to the traffic congestion problems suggested in the literature are presented as below:

\section{A. Expert System:}

Expert System based Traffic Light Controller described by Findler and Stapp (1992) ${ }^{[5]}$. An expert system uses the set of rules to decide the next action. In traffic Light control such an action can change some of control parameters that means the totally new system implementation required.

\section{B. Fuzzy Logic:}

Fuzzy Logic Traffic Light Controller described by Tan (1995). The fuzzy logic controller determines the time that the traffic light should stay in a certain state, before switching to the next state. This system has the disadvantage of the controller since it depends on the preset quantification values for fuzzy variables.

\section{Reinforcement Learning:}

Reinforcement learning based Traffic Light Controller described by Thorpe ${ }^{[4]}(1997)$. In this system, neural network is used for the traffic-light based value function which predicts the waiting time for all cars standing at the junction. This means that traffic light controller have to deal with a huge number of states, where learning time and variance may be quite large.

\section{Prediction based optimization:}

Prediction based optimization based Traffic Light Controller described by Tavladakis and Voulgaris ${ }^{[3]}$ (1999). In this system, Measurements taken during the current cycle are used to test several possible settings for the next cycle. Since it only uses the data of one cycle, it could not handle strong fluctuations in traffic flow well. Hence it gives poor performance.

\section{E. Using Magneto-Resistive Sensors:}

The author Cai Bai-gen ${ }^{[2]}$ (2009) design a vehicle detection system based on magneto-resistive sensor is composed by wireless traffic information collection nodes which are set on two sides of road to detect vehicle signal . The magneto-resistive sensor is costly and maintenance cost of the system will be more if the system fails. This system has lacks of emergence measures.

- The proposed operation of Traffic Light Controller uses the Infrared sensors to detect vehicle. The presence or absence of vehicle is sensed by sensor assembly mounted on each road. This act as input to Traffic Light Control Unit.

- The basic operation of Smart Traffic System Unit can be realized by using embedded system which has advantage of simplicity, user friendly, easy programmable.

- The smart system must inform the person about the happenings and should also inform alternate route to avoid loss of time. In this project, we are implementing to inform the car drivers about congested and alternate routes for rapid transit. Conditions on roads are communicated to car drivers on their personal GSM mobile sets, which will help them to select proper route.

- Proposed STC system is able to handle the problem of emergency vehicle by giving priority to it. That is, when emergency vehicle is passing by the route then other vehicles will get red signal and emergency vehicle can easily pass by the route.

\section{Proposed Model}

The Infrared Sensors mounted on road to detect the vehicle on the road. The presence or absence of a vehicle is sensed by a sensor assembly mounted on each road. This acts as an input to the STC unit. The STC unit generates an output signals for Red, Green and Yellow Signal and monitor their timings taking into consideration the number of vehicles on each road. The same information is transmitted to the mobile user which will request for congestion status. If vehicle driver at junction send SMS on GSM mobile phone to STC unit, the driver will get message indicating congestion status of road. STC system will also give information about alternate route to the user, if present traffic is heavy.

In addition to above, in the emergency mode, for a vehicle like ambulance, fire fighter or police car, the signals are altered for the fast and easy movement of these vehicle. If an emergency vehicle is passing by the 
route, the signals on the roads which are crossing this route will be immediately made red to stop vehicles on these routes. This is a very important feature which is very useful in case of emergency.

\subsection{Block Diagram}

The Fig.1. Shows block diagram of Smart Traffic Light Controller which consists of API, STC circuit, computer system, IR Sensors. The sensors detect traffic present at signal, and provide it as input to the computer system and then time period is decided through programming and this time is given to signal through control circuit. So finally depending on current traffic present at signal, time period is assign to it and the red, green, orange light signal glows accordingly.

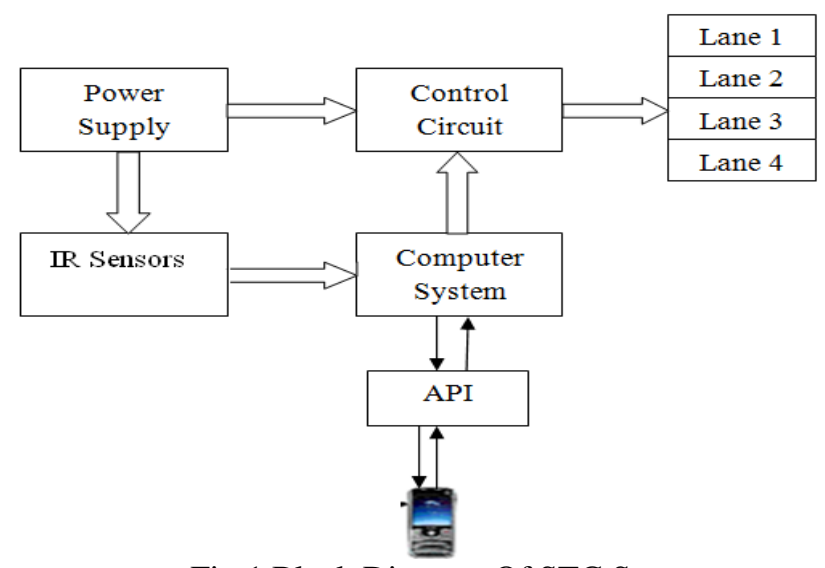

Fig.1.Block Diagram Of STC System

The system provides the information about the traffic present at different signal to the driver who wishes to get that information on their mobile phone.

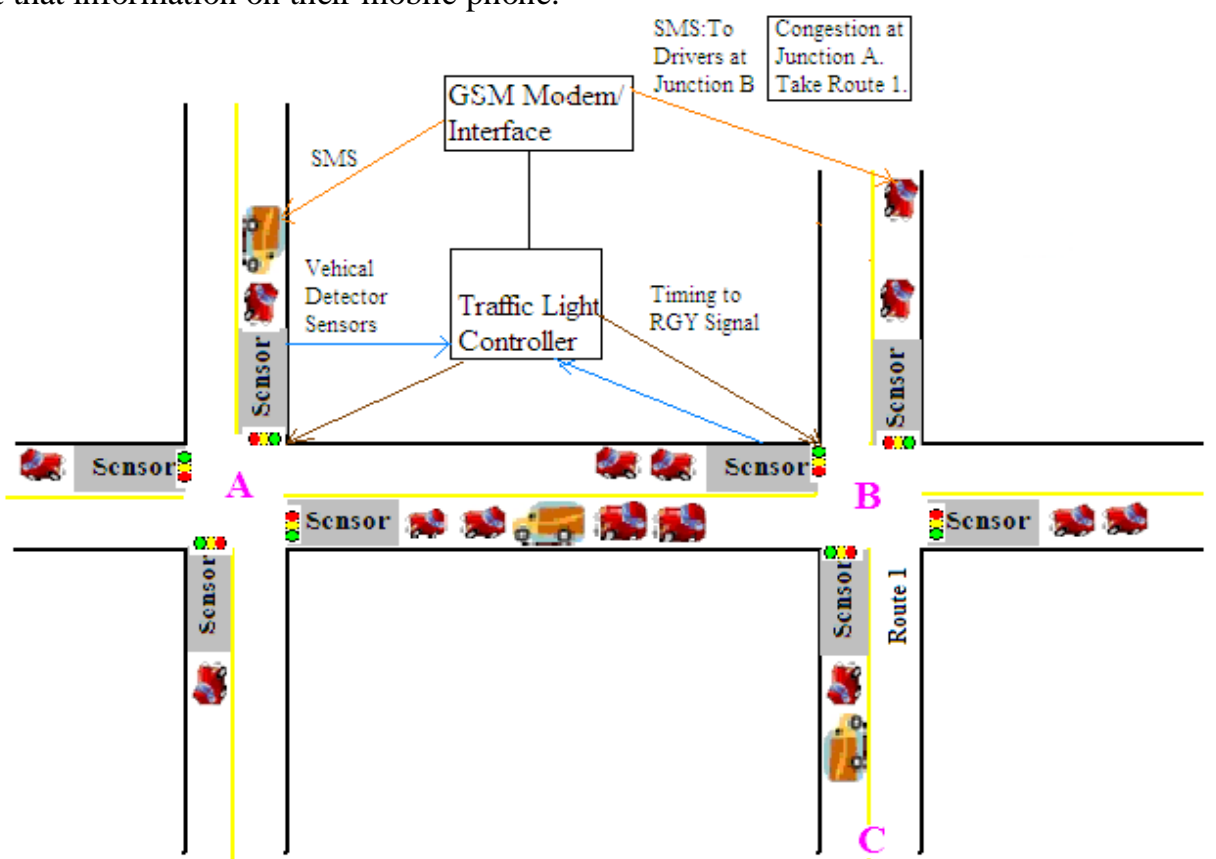

Fig.2. Basic concept of Smart Traffic Light Controller

The Basic Model of Smart Traffic Light Controller System is shown in Fig.2. In proposed model the junctions are shown by letter A, B and C. To detect traffic length the Infra-Red sensors are mounted on road side at each junction. Sensors detect the traffic level and provide this as input to computer system where it decides time period for each red, green and orange light for which it remains in glowing state. As shown in figure, if there is heavy traffic congestion at junction A then through SMS, car driver will be informed about congestion so that he can decide to take another route i.e. route 1 passing by $\mathrm{C}$ on their mobile phone ${ }^{[1]}$.

In addition to above, in the emergency mode, for a vehicle like ambulance, fire fighter or police car, the signals are altered for the fast and easy movement of these vehicle. Consider Fig.2, if an emergency vehicle 
is passing by the route $\mathrm{A}-\mathrm{B}$, the signals on the roads which are crossing this route will be immediately made red to stop vehicles on these routes. This is a very important feature which is very useful in case of emergency.

The basic operation of STC can be realized using embedded system and sensor network. In this model control circuit and computer system, parallel communication interface, API is used.

\subsection{Circuit Diagram}

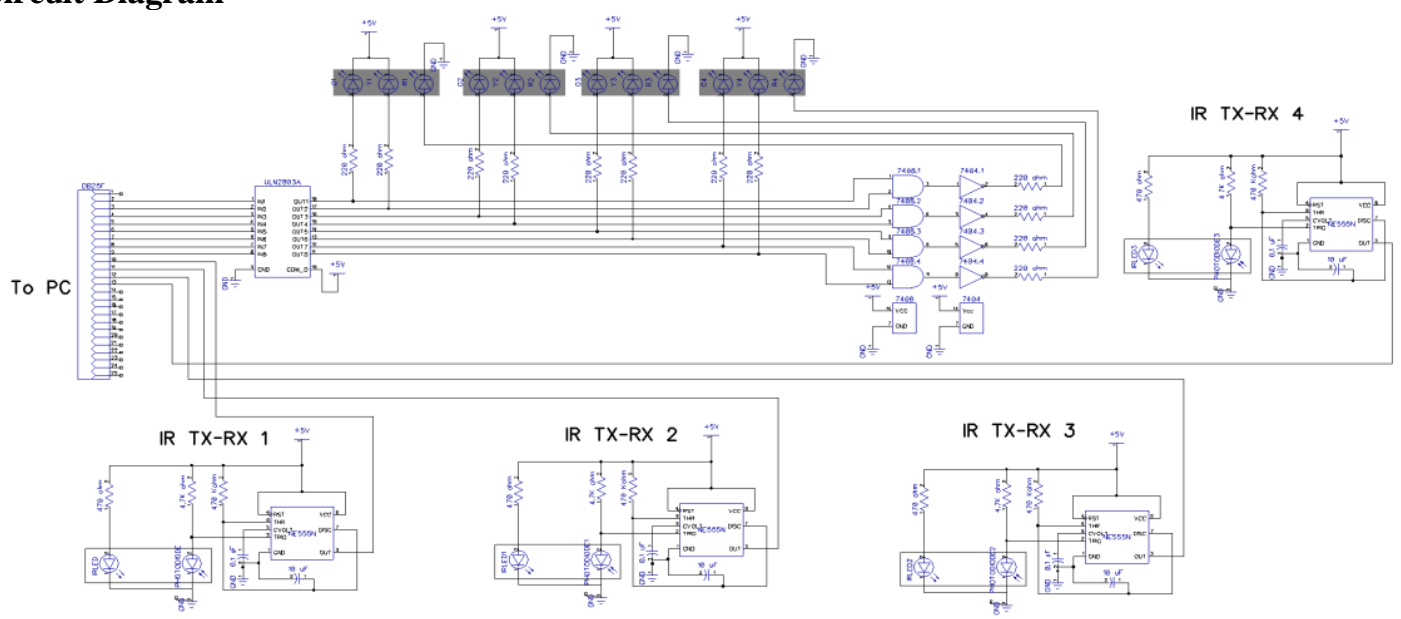

Fig.3. Circuit diagram of Smart Traffic Light Controller

This Circuit diagram consists of ULN 2803A, AND Gate IC 7408, NOT gate IC 7404, timer IC 555, IR Transmitter and Receiver. Pin 2-9 of parallel port are connected to input pins of ULN 2803A and output pins of ULN 2803A are connected to input of 4 AND gates. The ULN2803A is a high-voltage, high-current Darlington transistor array which is used for higher current capability.

Four green LEDs are connected with all even pins 12-14-16-18 and yellow LEDs are connected with all odd pins 11-13-15-17. The output of all AND gates are given to all four RED LEDs through NOT gates. The power supply to IC555 is given directly. IR LED and IR photo diode is used to detect laser beam.

\section{Conclusion}

The smartness of traffic signal controller is introduced in this project with powerful functions and hardware interface. The software API provides easy interface to the administrator.

Proposed system works efficiently over the present traffic controlling system in respect of less waiting time, efficient operation during emergency mode and suggesting alternate route.

\section{References}

[1] Shilpa S. Chavan(Walke), Dr. R. S. Deshpande, J. G. Rana (2009) "Design of Intelligent Traffic Light Controller using Embedded System", Second International Conference on emerging trends in Engineering and Technology.

[2] Cai Bai-gen, ShangGuan Wei, Wang Jian \& Chen Rui (2009) "The Research and Realization of Vehicle Detection System Based on Wireless Magneto-resistive Sensor", Second International Conference on Intelligent Computation Technology and Automation.

[3] Tavladakis and Voulgaris (1999) "Development of an autonomous adaptive traffic control system", In ESIT '99 - The European Symposium on Intelligent Techniques.

[4] Thorpe (1997) "Vehicle traffic light control using sarsa", Master's thesis, Department of Computer Science, Colorado State University.

[5] Findler and Stapp (1992) "A distributed approach to optimized control of street traffic signals", Journal of Transportation Engineering. 